\title{
Estresse ocupacional presente nas atividades da equipe de enfermagem em centro cirúrgico: Revisão integrativa
}

Occupational stress present in the activities of the nursing team in the operating room: Integrative review

Estrés ocupacional presente en las actividades del equipo de enfermería en el quirófano: Revisión integradora

Catarina Santos Ramos

ORCID: https://orcid.org/0000-0002-2376-8333

Centro Universitário Metropolitano da Amazônia, Brasil

E-mail: catramosblue@gmail.com

Irany Almeida Silva dos Santos

ORCID: https://orcid.org/0000-0002-0793-9307

Centro Universitário Metropolitano da Amazônia, Brasil

E-mail: iranyalmeida@hotmail.com

Ana Gracinda Ignácio Silva

ORCID: https://orcid.org/0000-0001-6439-7842

Centro Universitário Metropolitano da Amazônia, Brasil

E-mail: anagracinda08@gmail.com

Brenda Tanielle Dutra Barros

ORCID: https://orcid.org/0000-0002-3184-050X

Centro Universitário Metropolitano da Amazônia, Brasil

E-mail: brendatanielle.enf@gmail.com

Rômulo Leno Miranda Barros

ORCID: https://orcid.org/0000-0003-1802-4521

Universidade da Amazônia, Brasil

E-mail: romuloleno@hotmail.com

Rogério Oliveira Bailão

ORCID: https://orcid.org/0000-0003-3047-5077

Escola Superior da Amazônia, Brasil

E-mail: rogerio_bailao@hotmail.com

Tatiana Fabíola da Silva Lima

ORCID: https://orcid.org/0000-0001-5966-9727

Centro Universitário Metropolitano da Amazônia, Brasil

E-mail: tatiana.fs.lima2@gmail.com

Virgínia Mercês Lara Pessoa Oliveira

ORCID: https://orcid.org/0000-0002-4417-637X

Centro Universitário Metropolitano da Amazônia, Brasil

E-mail: virginiaoliveira@famaz.edu.br

Luceny Oliveira Mendes

ORCID: https://orcid.org/0000-0003-0505-9493

Centro Universitário Metropolitano da Amazônia, Brasil

E-mail: lumemetais08@hotmail.com

Elder Mescouto de Brito

ORCID: https://orcid.org/0000-0001-7246-2309

Centro Universitário Metropolitano da Amazônia, Brasil

E-mail: eldermescouto@outlook.com

Bianca Campos de Oliveira

ORCID: https://orcid.org/0000-0001-8144-8453

Universidade do Estado do Pará, Brasil

E-mail: biancaoliveira96142@gmail.com

Juliana Farias Barbosa

ORCID: https://orcid.org/0000-0002-8749-9451

Universidade da Amazônia, Brasil

E-mail: julianafarias783@gmail.com

Rosana Cristina Coqueiro Campos

ORCID: https://orcid.org/0000-0003-2719-7248

Centro Universitário Metropolitano da Amazônia, Brasil

E-mail: rosanacampos06@yahoo.com.br 


\author{
Katielem Melo Vale \\ ORCID: https://orcid.org/0000-0003-4868-1223 \\ Centro Universitário Metropolitano da Amazônia, Brasil \\ E-mail: katielem.enf@gmail.com \\ Ana Mara Franco Almeida Couto \\ ORCID: https://orcid.org/0000-0003-2871-6134 \\ Faculdade Paraense de Ensino, Brasil \\ E-mail: maraanafranco@gmail.com
}

\begin{abstract}
Resumo
O centro cirúrgico é uma unidade complexa, restrita, na qual a equipe se depara com diversas situações que podem ser percebidas como estressores. O estudo teve como objetivo descrever as evidências científicas encontradas na literatura científica do período de 2015 a 2020, sobre os fatores estressores e suas consequências, para os profissionais de enfermagem no trabalho do centro cirúrgico. Trata-se de um estudo de Revisão Integrativa de literatura, descritivo, de abordagem qualitativa. As bases de dados pesquisadas foram BVS, LILACS e SciELO. Para extração dos dados foi utilizado o formulário de Ursi \& Galvão. Para a análise crítica dos estudos foi aplicado o sistema de classificação por hierarquia das evidências. A amostra do estudo foi constituída de nove artigos originais. Os resultados foram apresentados e discutidos a partir de duas categorias temáticas: Fatores estressores do trabalho em centro cirúrgico e As consequências do estresse no trabalho em centro cirúrgico. O trabalho do enfermeiro inserido nas instituições de saúde é muitas vezes multifacetado, dividido e submetido à uma diversidade de cargos que geram desgaste. Considerou-se que a enfermagem é uma profissão estressante e isto se relaciona ao trabalho com pessoas que sofrem e requerem grande demanda de atenção. Concluiu-se que os fatores de estresse ocupacional são de natureza ambiental, social, profissional relacionados sobretudo a natureza das atividades desenvolvidas, quantitativo de profissionais, planejamento das atividades, materiais e equipamentos, relações interpessoais, são fatores que acarretam consequências físicas e psicológicas.
\end{abstract}

Palavras-chave: Enfermagem; Estressores; Estresse; Ocupacionais; Centro cirúrgico.

\begin{abstract}
The operating room is a complex, restricted unit, in which the team is faced with several situations that can be perceived as stressors. The study aimed to describe the scientific evidence found in the scientific literature from 2015 to 2020, about stressors and their consequences, for nursing professionals at work in the operating room. This is an integrative literature review study, descriptive, with a qualitative approach. The databases searched were BVS, LILACS and SciELO. For data extraction, the Ursi \& Galvão form was used. For the critical analysis of the studies, the classification system by hierarchy of evidence was applied. The study sample consisted of nine original articles. The results were presented and discussed from two thematic categories: Stress factors at work in the operating room and the consequences of stress at work in the operating room. Divided and submitted to a variety of positions that generate wear and tear. Nursing was considered to be a stressful profession and this is related to working with people who suffer and require a great deal of attention. It was concluded that the occupational stress factors are of an environmental, social, professional nature, mainly related to the nature of the activities developed, the number of professionals, the planning of activities, materials and equipment, interpersonal relationships, are factors that have physical and psychological consequences.
\end{abstract}

Keywords: Nursing; Stressors; Stress; Occupational; Surgery Center.

\title{
Resumen
}

El quirófano es una unidad compleja y restringida, en la que el equipo se enfrenta a diversas situaciones que pueden percibirse como estresantes. El estudio tuvo como objetivo describir la evidencia científica encontrada en la literatura científica de 2015 a 2020, sobre los estresores y sus consecuencias, para los profesionales de enfermería que laboran en el quirófano. Se trata de un estudio de revisión bibliográfica integradora, descriptiva, con enfoque cualitativo. Las bases de datos buscadas fueron BVS, LILACS y SciELO. Para la extracción de datos se utilizó el formulario de Ursi \& Galvão. Para el análisis crítico de los estudios se aplicó el sistema de clasificación por jerarquía de evidencia. La muestra de estudio estuvo formada por nueve artículos originales. Los resultados fueron presentados y discutidos a partir de dos categorías temáticas: Factores de estrés del trabajo en quirófano y las consecuencias del estrés laboral en el quirófano. El trabajo de las enfermeras insertas en las instituciones de salud, suele ser multifacético, dividido y sometido a una variedad de posiciones que generan desgaste. La enfermería se consideraba una profesión estresante y está relacionada con el trabajo con personas que sufren y requieren mucha atención. Se concluyó que los factores de estrés ocupacional son de carácter ambiental, social, profesional, principalmente relacionados con la naturaleza de las actividades desarrolladas, el número de profesionales, la planificación de actividades, materiales y equipos, las relaciones interpersonales, son factores que tienen efectos físicos y consecuencias psicológicas.

Palabras clave: Enfermería; Factores estresantes; Estrés; Ocupacional; Centro cirúrgico. 


\section{Introdução}

O Centro Cirúrgico (CC) apresenta-se como uma área restrita, com carga de trabalho excessiva, em proporção inadequada ao número de profissionais presentes da área da enfermagem com qualificação, turnos rotativos, baixa remuneração, risco biológico inerente à manipulação de pacientes em período Peri operatório, o risco físico determinado pelo uso de Raios-X nas salas cirúrgicas, o trabalho em postura inadequada, em pé e o uso de novas tecnologias sem o devido preparo (Jacques et al., 2015).

Entende-se por fator estressor todo estímulo que ocasione uma resposta ou reação ao estressor. O estresse é considerado uma patologia ocupacional crescente, com especial incidência no setor dos serviços e um maior risco em atividades que exigem dedicação personalizada, como é visto nos serviços de enfermagem (Puerto, 2017).

O risco para o surgimento do estresse ocupacional não é descartado e pode acometer qualquer profissional, de qualquer área, incluindo o enfermeiro. Pontua-se que dentro dos hospitais, existe uma alta demanda de serviços e, devido a isto, surge a problemática do cansaço intenso da equipe atuante, que reflete no desempenho profissional e aciona o desgate psico-corporal (Versa et al., 2012).

O trabalho do enfermeiro no CC tem se tornado cada vez mais complexo, na medida em que há a necessidade de integração entre as atividades que abrangem a área técnica, gerencial, administrativo-burocrática, assistencial, de ensino e pesquisa, e na dimensão de sua atuação, por ser um profissional que atua diretamente com uma equipe diversificada profissionalmente (Gomes \& Dutra, 2014).

O CC é uma unidade hospitalar marcadamente de intervenções invasivas e de recursos materiais com alta precisão e eficácia, requer profissionais habilitados para atender diferentes necessidades do usuário diante da elevada densidade tecnológica à variedade de situações que lhe conferem uma dinâmica peculiar de assistência em saúde. É, portanto, considerado como cenário de alto risco, onde os processos de trabalho constituem-se em práticas complexas, interdisciplinares, com forte dependência da atuação individual e da equipe em condições ambientais dominadas por pressão e estresse (Martins \& Dall'agnol, 2016).

Além disso, profissionais com enfermeiros, técnicos e auxiliares de enfermagem do CC vêm suportando cargas de trabalho cada vez maiores, com proporção quantitativa inadequada de profissional para cada paciente, rotatividade de turnos, manipulação de substâncias tóxicas e presença de condições que tragam riscos pertinentes ao ambiente, o que leva a uma excessiva sobrecarga no ambiente em que atua, podendo resultar em transtornos físicos e psicológicos, afetando e comprometendo sua saúde e qualidade de vida (Murano, Santos \& Beresin, 2009).

Estudar a problemática é relevante, pois é crescente o interesse nos últimos anos pelo estudo do estresse ocupacional e dos fatores estressores no local de trabalho devido aos impactos que podem ter na saúde dos trabalhadores (Puerto et al., 2017).

Diante desse cenário, questiona-se: Quais os fatores estressores e suas consequências no trabalho de enfermagem em centro cirúrgico, são evidenciados na literatura científica de 2015 a 2020 ?

O presente estudo teve como objetivo descrever as evidências científicas encontradas na literatura científica do período de 2015 a 2020, sobre os fatores estressores e suas consequências, para os profissionais de enfermagem em seu trabalho do centro cirúrgico.

\section{Metodologia}

Trata-se de um estudo de Revisão Integrativa de literatura, descritivo, de abordagem qualitativa. Os estudos de revisão têm a função de demonstrar de forma clara e abrangente as publicações científicas, baseando-se em conceitos diversos 
e em distintos saberes (Lima et al., 2021). Se tratando de pesquisas qualitativas, a grande questão que as envolvem são a autonomia da interpretação dos estudos, por parte do autor a partir de uma nova questão posta em pauta (Pereira et al., 2018).

Para a construção deste trabalho seguiu-se as seis fases do processo de elaboração de Revisão Integrativa de Literatura: $1^{\circ}$ Etapa - identificação da temática e seletiva das hipóteses ou questões de pesquisa. $2^{\circ}$ Etapa - instauração de critérios para inclusão e exclusão de estudos/amostragem ou investigação na literatura. $3^{\circ}$ Etapa - escolha dos dados a serem coletados das pesquisas eleitas/categorização dos estudos. $4^{\circ}$ Etapa -julgamento crítico da literatura incluída na revisão. $5^{\circ}$ Etapa - interpretação das informações. $6^{\circ}$ Etapa - exposição da revisão/resumo do conhecimento (Teixeira et al., 2014).

Nesse sentido, na $1^{\text {a }}$. Etapa foi definido a seguinte pergunta norteadora: Quais os fatores estressores e suas consequências no trabalho de enfermagem em centro cirúrgico, são evidenciados na literatura científica de 2015 a 2020 ?

Esta questão seguiu a lógica da estratégia PICO adaptada, seguindo a estruturação recomendada para estudos qualitativos em que o P corresponde aos participantes, o I ao fenômeno de interesse e CO ao contexto do estudo (Cardoso et al., 2019).

Neste estudo, o "P", relaciona-se aos profissionais da enfermagem (enfermeiros/as, técnicos e auxiliares de enfermagem), o "I", relaciona-se aos motivos estressores e suas consequências no trabalho de enfermagem; o "CO", fatores estressores e suas consequências no trabalho de enfermagem do centro cirúrgico, evidenciados na literatura nos últimos cinco anos (Mombaque \& Secoli, 2018).

Na segunda etapa a busca de evidências foi realizada nas bases Biblioteca Virtual em Saúde (BVS), Literatura Latino-Americano e do Caribe em Ciências da Saúde (LILACS), Scientific Electronic Library Online (SciELO). Referentes ao período de 2015 a 2020 nacionais, por meio das palavras-chave "Enfermagem", "estressores", "estresse" "ocupacionais" "centro cirúrgico". Como estratégia de busca, foi utilizado o cruzamento dos descritores com utilização dos operadores booleanos "And"; "Or".

Como critérios de inclusão foram definidos: Artigos originais completos referentes ao período de 2015 a 2020, na língua portuguesa e com abordagem ao tema de estudo. Foram excluídos textos incompletos, resumos, língua estrangeira, publicações fora do período definido, sem afinidade com o tema, monografias, trabalhos de conclusão de curso e artigos de revisão teórica.

Na terceira etapa, da extração de dados foi utilizado o formulário Ursi \& Galvão (2005) para coleta de dados relevantes para o estudo.

$\mathrm{Na}$ quarta etapa foi realizado a análise crítica dos estudos aplicando o sistema de classificação de evidências, adotando-se a hierarquia das evidências, em relação ao delineamento de pesquisa. (Soares et al., 2014): Nível 1: Evidências resultantes da meta-análise; Nível 2: Evidências com delineamento experimental; Nível 3: Evidências de estudo quaseexperimental; Nível 4: Evidências de estudo não-experimental ou com abordagem qualitativa; Nível 5: Evidências de relatos de casos ou de experiência; Nível 6: Evidências com opiniões de especialistas.

Nesta quinta etapa, apresentam-se os resultados da pesquisa, onde as principais evidências foram elencadas em quadros sínteses.

E na sexta etapa, foi elaborado e apresentado a definição do desfecho obtido no estudo a partir das seguintes categorias temáticas: Fatores estressores do trabalho em centro cirúrgico e as Consequências do estresse neste trabalho.

\section{Resultados}

A amostra final do estudo foi de nove artigos que atenderam ao objeto do estudo proposto. Esse processo de inclusão, elegibilidade, seleção e identificação dos estudos foi disposto no Fluxograma 1 PRISMA. 
Fluxograma 1. Registro dos estudos selecionados.

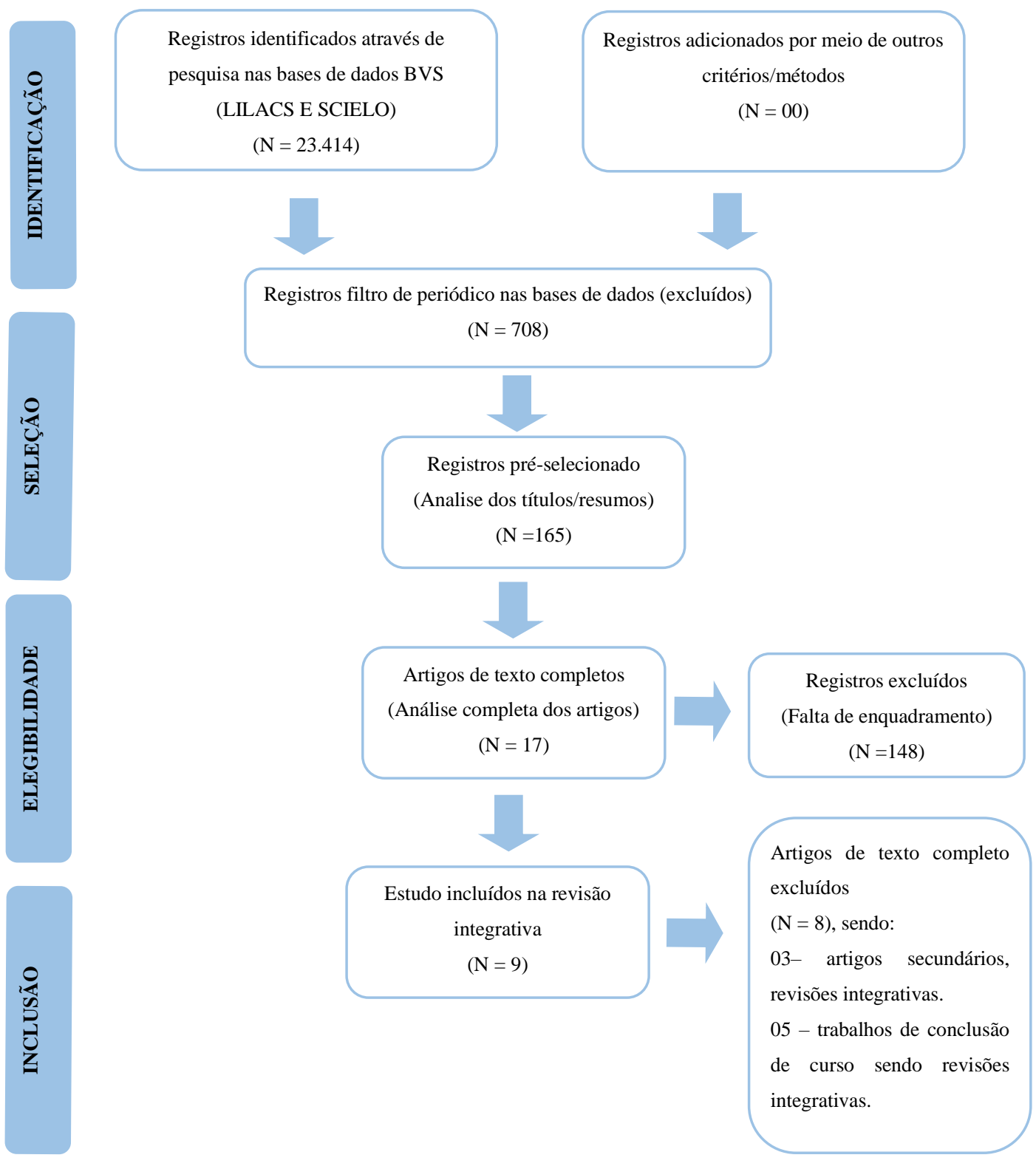

Fonte: Adaptado de Moher et al. (2009).

Os principais artigos e as principais evidências foram sintetizadas e caracterizadas em quadros sínteses para encontrar os nexos entre os artigos pesquisados, encontrando resposta à questão desta pesquisa, e objetivos propostos. Posteriormente, foi comparado ao referencial teórico que fundamentou o estudo. Para tal, foi realizado análise de conteúdo contendo Título do artigo, Base de dados, Revista/ano, Local da pesquisa e Objetivos, como pode ser observado no Quadro-síntese 1: 
Quadro-síntese 1. Caracterização dos artigos.

\begin{tabular}{|c|c|c|c|c|}
\hline TÍTULO DO ARTIGO & BASE DE DADOS & REVISTA/ANO & LOCAL DA PESQUISA & OBJETIVOS \\
\hline $\begin{array}{l}\mathrm{O} \text { estresse da equipe de } \\
\text { enfermagem no centro } \\
\text { cirúrgico }\end{array}$ & SCIELO & RIES, ISSN - 2016 & $\begin{array}{l}\text { Hospital privado do } \\
\text { Extremo Sul Catarinense }\end{array}$ & $\begin{array}{l}\text { Identificar quais são os } \\
\text { possíveis fatores causadores } \\
\text { de estresse da equipe de } \\
\text { enfermagem atuante no } \\
\text { Centro Cirúrgico }\end{array}$ \\
\hline $\begin{array}{l}\text { Qualidade de vida dos } \\
\text { profissionais atuantes no } \\
\text { centro cirúrgico. }\end{array}$ & SCIELO & $\begin{array}{l}\text { VER. PSICOL SAÚDE E } \\
\text { DEBATE- } 2017\end{array}$ & 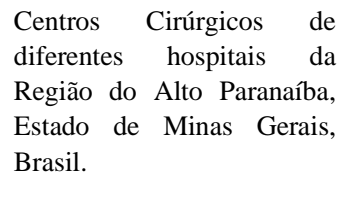 & $\begin{array}{l}\text { Verificar quais fatores de } \\
\text { estresse podem interferir na } \\
\text { qualidade de vida dos } \\
\text { trabalhadores do Centro } \\
\text { Cirúrgico de diferentes } \\
\text { hospitais }\end{array}$ \\
\hline $\begin{array}{l}\text { Estressores e sintomas de } \\
\text { estresse vivenciados por } \\
\text { profissionais em um centro } \\
\text { cirúrgico. }\end{array}$ & LILACS & $\begin{array}{l}\text { REME- Revista Mineira de } \\
\text { Enfermagem -2010 }\end{array}$ & $\begin{array}{lll}\text { Hospital geral- } & \text { Passo } \\
\text { Fundo-RS. } & & \end{array}$ & $\begin{array}{l}\text { Identificar quais estresses e } \\
\text { sintomas os profissionais da } \\
\text { saúde estão vivenciando em } \\
\text { um centro cirúrgico. }\end{array}$ \\
\hline $\begin{array}{l}\text { Síndrome de burnout entre } \\
\text { enfermeiros de um hospital } \\
\text { universitário }\end{array}$ & SCIELO & Rev baiana enferm - 2018 & $\begin{array}{l}\text { Hospital universitário } \\
\text { público da Região Sul do } \\
\text { Brasil }\end{array}$ & $\begin{array}{l}\text { Identificar quais os níveis da } \\
\text { síndrome de Burnout } \\
\text { presente nos enfermeiros de } \\
\text { um hospital universitário. }\end{array}$ \\
\hline $\begin{array}{l}\text { Geradores de estresse para } \\
\text { os trabalhadores de } \\
\text { enfermagem de centro } \\
\text { cirúrgico }\end{array}$ & LILACS & $\begin{array}{l}\text { Semina: Ciências Biológicas } \\
\text { e da Saúde, Londrina - } 2015\end{array}$ & $\begin{array}{l}\text { Hospital Universitário do } \\
\text { estado do Paraná. }\end{array}$ & $\begin{array}{l}\text { Identificar quais fatores } \\
\text { contribuem para existir o } \\
\text { estresse entre trabalhadores } \\
\text { de enfermagem de Centro } \\
\text { Cirúrgico de um hospital } \\
\text { público de grande porte e } \\
\text { alta complexidade }\end{array}$ \\
\hline $\begin{array}{l}\text { Dualidade entre satisfação e } \\
\text { Sofrimento no trabalho da } \\
\text { equipe de enfermagem em } \\
\text { centro cirúrgico }\end{array}$ & SCIELO & Rev. SOBECC - 2017 & $\begin{array}{l}\text { Hospital de médio porte da } \\
\text { Região Noroeste do Paraná }\end{array}$ & $\begin{array}{l}\text { Apreender a percepção da } \\
\text { equipe de Enfermagem } \\
\text { sobre qual a relação entre } \\
\text { trabalho em centro cirúrgico } \\
\text { (CC) e saúde. }\end{array}$ \\
\hline $\begin{array}{l}\text { Qualidade de vida dos } \\
\text { profissionais de enfermagem } \\
\text { atuantes em setores críticos }\end{array}$ & SCIELO & Revista Cuidarte. 2018 & $\begin{array}{l}\text { Hospital privado do } \\
\text { Extremo Sul Catarinense }\end{array}$ & $\begin{array}{l}\text { Analisar a qualidade de vida } \\
\text { de profissionais de } \\
\text { enfermagem atuantes em } \\
\text { setores críticos. }\end{array}$ \\
\hline $\begin{array}{l}\text { Qualidade de vida no } \\
\text { trabalho da equipe de } \\
\text { enfermagem do centro } \\
\text { cirúrgico. }\end{array}$ & LILACS & Enferm. Foco 2018 & $\begin{array}{l}\text { Hospital de referência em } \\
\text { Teresina (PI). }\end{array}$ & $\begin{array}{l}\text { Avaliar a qualidade de vida } \\
\text { presente no trabalho da } \\
\text { equipe de enfermagem do } \\
\text { centro cirúrgico. }\end{array}$ \\
\hline $\begin{array}{l}\text { Satisfação profissional da } \\
\text { equipe de enfermagem do } \\
\text { centro cirúrgico em hospital } \\
\text { público de média } \\
\text { complexidade. }\end{array}$ & SCIELO & $\begin{array}{l}\text { Rev enferm UFPE on line- } \\
2016\end{array}$ & $\begin{array}{l}\text { Hospital público do Norte } \\
\text { Paraná/PR. }\end{array}$ & $\begin{array}{l}\text { Fazer a verficação da } \\
\text { satisfação profissional de } \\
\text { trabalhadores }\end{array}$ \\
\hline
\end{tabular}

Fonte: Adaptado de Ursi \& Galvão (2015).

Neste estudo a principal evidência foi de nível 4, com 3 artigos de abordagem qualitativa e 6 de abordagem quantitativa, como pode ser observado nas contribuições do Quadros-síntese 2, a seguir: 
Quadro-síntese 2. Contribuições dos estudos.

\begin{tabular}{|c|c|c|}
\hline ARTIGO & $\begin{array}{c}\text { NÍVEL DE } \\
\text { EVIDENCIA }\end{array}$ & RESULTADOS \\
\hline $\begin{array}{l}\text { O estresse da equipe de } \\
\text { enfermagem no centro } \\
\text { cirúrgico }\end{array}$ & 4 & $\begin{array}{l}\text { Quanto a sintomatologia } 69,56 \% \text { dos profissionais do Centro Cirúrgico relatam tensão muscular; } 67,39 \% \\
\text { ansiedade e cefaléia; } 65,21 \% \text { dores lombar; } 45,65 \% \text { alopécia; } 43,47 \% \text { irritabilidade generalizada; } 41,30 \% \\
\text { diminuição da libido e impulso sexual; } 32,60 \% \text { desordens respiratórias; } 30,43 \% \text { erupções na pele, falta de } \\
\text { concentração, prejudicada e azia; } 28,26 \% \text { problemas urinários e mialgia; } 26,08 \% \text { gases e bruxismo; } 23,91 \% \\
\text { resfriados de longos períodos, insônia, sensação de palpitação; } 21,73 \% \text { doença osteomuscular relativa ao } \\
\text { trabalho (DORT) e disfunção dentária; } 19,56 \% \text { gastrite; } 17,39 \% \text { autoconceito reduzido; } 15,21 \% \text { enjoos e } \\
\text { isolamento social; } 13,04 \% \text { facilidade em adquirir a doenças infecciosas; } 10,86 \% \text { falta do interesse da aparência } \\
\text { social e baixa atividade imunológica; } 8,69 \% \text { extremidades mal oxigenadas e úmidas e hipertensão arterial; } \\
6,52 \% \text { depressão, disfagia e diarréia psicogênica; } 4,34 \% \text { mau humor; } 2,17 \% \text { desmotivação, refluxo estomacal, } \\
\text { asma, hipotireoidismo, inflamação do duodeno. }\end{array}$ \\
\hline $\begin{array}{l}\text { Qualidade de vida dos } \\
\text { profissionais atuantes } \\
\text { no centro cirúrgico. }\end{array}$ & 4 & $\begin{array}{l}\text { A condição que mais causa estresse entre os profissionais, segundo o estudo, é o tipo de ambiente sendo que os } \\
\text { outros fatores podem variar conforme a categoria profissional. Em relação aos técnicos de enfermagem, o } \\
\text { segundo fator elencado que mais causa estresse é a sobrecarga de trabalho }(28,6 \%) \text {, relações interpessoais } \\
\text { (19\%), pouca de assiduidade e pontualidade }(11,9 \%) \text {, e falta de recursos materiais }(9,5 \%) \text {, não se tendo citado a } \\
\text { baixa remuneração e desvalorização. Referente aos enfermeiros, as relações entre a equipe apresentaram a } \\
\text { mesma proporção do tipo de ambiente (24\%), seguido da falta de recursos materiais/insumos, pouca assiduidade } \\
\text { e pontualidade, sobrecarga de trabalho (16\%), e baixa remuneração e desvalorização (4\%). }\end{array}$ \\
\hline $\begin{array}{l}\text { Estressores e sintomas } \\
\text { de estresse vivenciados } \\
\text { por profissionais em um } \\
\text { centro cirúrgico. }\end{array}$ & 4 & $\begin{array}{l}\text { Os profissionais acabam vivenciando variados fatores de estresse, os quais estão relacionados às relações } \\
\text { interpessoais, falta ou asência de recursos materiais, quantidade insuficiente de profissionais qualificados, alta } \\
\text { demanda de cirurgias e a própria condição de saúde dos pacientes. }\end{array}$ \\
\hline $\begin{array}{l}\text { Síndrome de burnout } \\
\text { entre enfermeiros de } \\
\text { um hospital } \\
\text { universitário }\end{array}$ & 4 & $\begin{array}{l}\text { Os enfermeiros participantes da pesquisa obtiveram níveis moderados de burnout para as três subescalas: } \\
\text { exaustão emocional, despersonalização e realização pessoal. Enfermeiros atuantes do CC apresentaram índice } \\
\text { alto na subescala exaustão emocional. }\end{array}$ \\
\hline $\begin{array}{l}\text { Geradores de estresse } \\
\text { para os trabalhadores de } \\
\text { enfermagem de centro } \\
\text { cirúrgico }\end{array}$ & 4 & $\begin{array}{l}\text { Foram evidenciados nas falas, que os fatores que levam à sobrecarga de trabalho e causam estresse são: pouco } \\
\text { tempo para a realizar atividades laborais que exigem que o trabalhador as realize com grande rapidez, além do } \\
\text { quantitativo reduzido de profissionais capacitados, exigindo que o profissional seja escalado consecutivamente à } \\
\text { mesma atividade. As falas evidenciam que a falta de planejamento das atividades a serem desenvolvidas, falta } \\
\text { de materiais, de recursos humanos e de equipamentos, são fatores que os levam ao estresse. Nos relatoss desta } \\
\text { pesquisa mostra-se que a interelação dos profissionais com o ambiente de trabalho pode acarretar em reações } \\
\text { que levem ao surgimento do estresse. Os profissionais de enfermagem experenciam o estresse relacionado com: } \\
\text { a alta carga trabalhista, ausência de planejamento das atividades, dos recursos humanos e dos insumos e o } \\
\text { trabalhar em um ambiente fechado. }\end{array}$ \\
\hline $\begin{array}{l}\text { Dualidade entre } \\
\text { satisfação e sofrimento } \\
\text { no trabalho da equipe } \\
\text { de enfermagem em } \\
\text { centro cirúrgico }\end{array}$ & 4 & $\begin{array}{l}\text { Questões que geram satisfação quanto à subjetividade do trabalhador referem-se ao grande valor dado ao } \\
\text { trabalho. Questões referentes ao processo de trabalho como: relações entre os profissionais que sejam positivas } \\
\text { acompanhadas pelo companheirismo dos colegas de trabalho, também geram satisfação. Aspectos relativos ao } \\
\text { ambiente também geram satisfação quando os participantes os entendem-se como geradores de conhecimento e } \\
\text { aprendizado no trabalho em CC, algumas vezes, são causadores de insatisfações que versão sobre as } \\
\text { características institucionais, que podem ser exemplificadas pela não valoração profissional, cobrança para ser } \\
\text { produtivo, pouco recursos humanos, grande carga de trabalho e a falta de tempo. Quanto às características do } \\
\text { processo de trabalho e específicas do ambiente de CC também são notados de forma negativa: a relação de } \\
\text { poder entre as diferentes categorias profissionais em CC. A equipe de Enfermagem vê no CC um ambiente } \\
\text { rodeado de riscos ocupacionais. Algumas circunstâncias do trabalho impactam em sua condição física, } \\
\text { produzindo dores osteomusculares, cansaço, artrite, artrose e cefaleia. Elencou-se a influência do trabalho nas } \\
\text { questões psicossociais, consequentes do estresse, ansiedade, irritabilidade. Insatisfação por não trabalhar, } \\
\text { dificuldade de interação e remuneração insatisfatória. }\end{array}$ \\
\hline $\begin{array}{c}\text { Qualidade de vida no } \\
\text { trabalho da equipe de } \\
\text { enfermagem do centro } \\
\text { cirúrgico. }\end{array}$ & 4 & $\begin{array}{l}\text { Este estudo pode mostrar que o trabalho no centro cirúrgico apresenta certo impacto na qualidade de vida dos } \\
\text { profissionais. Apesar de ter apresentado menor impacto na qualidade de vida no trabalho, a questão psicológica } \\
\text { mais significativa. Estas condições e os benefícios ofertados pela instituição e as condutas de responsabilidade } \\
\text { social, tratamento igualitário entre funcionários, prazer em trabalhar na organização, participação em decisões, } \\
\text { compromisso/responsabilidade, qualificação e o ato de solidariedade entre os profissionais no serviço são } \\
\text { questões que acabam interferindo na saúde mental destes trabalhadores. Este grade impacto psicológico pode ser } \\
\text { justificado devido grande parte dos dos profissionais possuírem mais de um vínculo }\end{array}$ \\
\hline $\begin{array}{l}\text { Satisfação profissional } \\
\text { da equipe de } \\
\text { enfermagem do centro } \\
\text { cirúrgico em hospital } \\
\text { público de média } \\
\text { complexidade. }\end{array}$ & 4 & $\begin{array}{l}\text { Os profissionais enfermeiros e técnicos de enfermagem trouxeram a autonomia como o componente de maior } \\
\text { importância vinculada à satisfação profissional. Observou-se visões distintas entre enfermeiros e técnicos } \\
\text { entrevistados. Os enfermeiros elencaram a questão financeira como componente de maior satisfação e a relação } \\
\text { com a equipe de enfermagem como último fator. Para os técnicos de enfermagem os requisitos de trabalho } \\
\text { trazem maior satisfação, já a autonomia profissional não possui a mesma representatividade para eles. As } \\
\text { normas institucionais foram apontadas como questão de menor importância para a categoria dos enfermeiros. A } \\
\text { categoria técnicos de enfermagem trouxe o status profissional como o fator de menor importância à satisfação } \\
\text { Em relação a satisfação percebida, a remuneração ocupou o primeiro lugar para os enfermeiros. }\end{array}$ \\
\hline $\begin{array}{l}\text { Qualidade de vida dos } \\
\text { profissionais de } \\
\text { enfermagem atuantes } \\
\text { em setores críticos }\end{array}$ & 4 & $\begin{array}{l}\text { Como resultados, apontou-se que a maioria dosentrevistados era do sexo feminino }(78,7 \%) \text {, com idade média de } \\
30 \text { anos, casados }(53,3 \%) \text { e com escore total elevado de qualidade de vida }(73,33) \text {. O domínio Relações Sociais } \\
\text { foi o mais bem avaliado e o que obteve o pior escore foi o domínio Físico. }\end{array}$ \\
\hline
\end{tabular}


Fonte: Adaptado de Ursi \& Galvão (2015).

Uma das formas para se analisar as informações obtidas de um estudo qualitativo, tem relação direta com a diversidade de achados das revisões. Compreende a diminuição, demonstração, conclusão de dados (Soares et al., 2014).

Os objetivos dos estudos que compuseram esta revisão foram predominantemente, e de modo geral, focados em identificar fatores estressores do trabalho em centro cirúrgico, e medir esses níveis de estresse, assim como relacionar o trabalho no centro cirúrgico a qualidade de vida e satisfação profissional.

A maioria da amostra dos artigos foram de profissionais técnicos de enfermagem seguido de enfermeiros predominantemente mulheres, apresentando um média de idade de 40 anos, casadas e que atuam em centro cirúrgico por um período entre 10 a 30 anos. Os resultados serão descritos a partir de duas categorias temáticas evidenciadas a partir da análise dos artigos: Fatores estressores do trabalho em centro cirúrgico e as Consequências do estresse no trabalho em centro cirúrgico.

\section{Discussão}

\section{Tema 1: Fatores estressores do trabalho em centro cirúrgico}

Neste tema, evidenciou-se que o fator de estresse inicia pela organização ambiental do centro cirúrgico, que se configura por ser ambiente fechado, dificultando a orientação em relação ao tempo, a comunicação com outras pessoas, causando a sensação de que estão alheios ao que se passa na instituição (Soares, et al., 2017; Stumm et al, 2010).

Entre os fatores estressores mais citados nos artigos e mais citados pelos enfermeiros, destacam-se: a alta carga de trabalho, redução ou ausência de compromisso com horários e a relação entre os profissionais que pertencem à equipe cirúrgica e pacientes; falta ou deficiência de recursos materiais, assim como sua má qualidade; salários baixos e valorização profissional; recursos humanos qualificados insuficientes; elevada demanda de cirurgias e a própria condição dos pacientes; intercorrências e as urgências; ausência de um correto planejamento e mudanças nos tipos de cirurgias e escala de sala (Soares, et al., 2017; Stumm et al, 2010; Jacques et al, 2015; Tostes et al., 2017; Souza et al, 2018).

Entre os fatores estressores mais citados pelo profissional técnico em enfermagem estão a carga exacerbada de trabalho, a relação com a equipe, falta de compromisso da equipe de cirurgia e dos pacientes, falta de recursos materiais. Os artigos não referenciaram a questão da remuneração e pouca valoração deste profissional. Entretanto, tanto enfermeiros quanto técnicos de enfermagem concordam que o ambiente fechado do centro cirúrgico é um fator de estresse ocupacional (Soares, et al., 2017; Stumm et al , 2010; Jacques et al, 2015; Tostes et al 2017).

Um estudo de satisfação profissional demonstrou que técnicos e enfermeiros reclamam da da falta de autonomia, porém os enfermeiros trazem como problemática a questão da relação entre satisfação profissional com a remuneração, enquanto para os técnicos de enfermagem, o que gera maior satisfação e a autonomia profissional não tem o mesmo peso. Já o fator status profissional foi o de menor importância para essa categoria (Paglione et al., 2016).

No que se refere as questões de qualidade de vida desses profissionais, pôde-se constatar que as condições ofertadas no trabalho, assim como os plantões e seus turnos, variadas tarefas, relações interpessoais, são considerados fatores estressores que podem impactar na saúde mental dos mesmos e impacto psicológico maior está relacionado aos vários vínculos empregatícios (Carvalho et al., 2018).

A relação com o profissional médico é outro ponto que merece atenção, sendo esta difícil, visto a cobrança e pouco reconhecimento frente ainda, ao crescente número de cirurgias sem aumento do número de funcionários, o que repercute na relação com o cliente; todos esses fatores são indutores de grande pressão psicológica e física, já que é um local onde não se 
admite erros pela própria natureza do serviço. Dessa forma, a equipe se percebe em um meio rodeado de riscos ocupacionais que afetam o eixo biopsicossocial (Tostes et al, 2017).

\section{Tema 2- Consequências do estresse no trabalho em centro cirúrgico}

O estresse ocupacional a que a equipe de enfermagem é submetida durante seu trabalho traz como consequências vários sintomas físicos e emocionais. Entre essas consequências foi evidenciado que vários sintomas físicos acometem esses profissionais, entre eles: problemas osteomusculares, cefaleia; alopécia, problemas psicossociais, redução da libido e impulso sexual; erupções cutâneas problemas gastrointestinais, problemas respiratórios e do aparelho urinário, bruxismo, sensação de palpitação cardíaca, baixa atividade imunológica, extremidades frias e úmidas e hipertensão arterial, asma, hipotireoidismo, cansaço; artrite e artrose; fibromialgia cervical. Além dos riscos por movimentos repetitivos, as perfurações acidentais e a radioatividade (Raio-X) (Soratto et al., 2016; Tostes et al., 2017).

Ainda é comum esses profissionais sentirem-se exaustos, o que foi mostrado em um estudo sobre a síndrome de burnout entre enfermeiros, onde os enfermeiros do centro cirúrgico apresentaram índice elevado na subescala exaustão emocional. Evidenciaram ainda, a ansiedade e o estresse gerados pelos fatores estressores que tem de conviver no dia a dia e pela responsabilidade de fazer tudo rápido e sem poder errar, além do que a proximidade cotidiana favorece desentendimentos que repercutem nas condições do sono desses profissionais e de seu bem-estar (Soratto et al., 2016; Santos et al., 2019; Tostes et al., 2017; Carvalho et al., 2018).

Nesse sentido, nota-se que as relações sociais estabelecidas no ambiente ocupacional do centro cirúrgico influenciam diretamente na saúde física e mental desses trabalhadores, sendo este, um ambiente gerador de conhecimento e aprendizado constante, fazendo com que esses profissionais vejam o centro cirúrgico como um trabalho que gostam, parecido como sua segunda casa e um setor importante onde trabalham em equipe e companheirismo (Tostes et al., 2017; Soares et al., 2017).

O Tema 1, evidencia os fatores estressores no trabalho do centro cirúrgico, e é diretamente proporcional ao local de trabalho, que se configura como um ambiente fechado, a alta carga de atividades, as relações entre os profissionais, falta de compromisso e falta de insumos; má remuneração e valorização profissional; número insuficiente de profissionais qualificados; elevada demanda de cirurgias; condição dos pacientes; intercorrências e urgências; ausência de um plano; turnos ou regime de plantões; variedade de tarefas; autonomia entre outros (Soares et al., 2017; Stumm et al., 2010; Jacques et al., 2015; Tostes et al 2017; Carvalho et al., 2018).

$\mathrm{Na}$ área da saúde, vários estudos têm se posicionado no que tange a questão do estresse que a equipe vivencia no dia-a-dia. Dentre os vários setores do ambiente hospitalar, o Centro Cirúrgico é um dos ambientes mais complexos do hospital, e onde, frequentemente, os seus trabalhos são desenvolvidos em um clima de tensão, pela existência de procedimentos estressantes geradores de ansiedade, quer pela gravidade dos pacientes, quer pela complexidade dos atos anestésicos e cirúrgicos (Passos, Silva \& Carvalho, 2010).

Além do exposto, pôde-se observar que os enfermeiros acabam enfrentando diversos problemas no trabalho como o estresse, o sofrimento e a morte de pacientes. Ainda, a carga de trabalho, a pressão dos companheiros, as atitudes ofensivas, o fato de lidar com novas tecnologias, seu comprometimento e, por vezes, não há o reconhecimento com as atividades como motivos estressantes comuns no cotidiano desses profissionais (Ueno et al., 2017).

Ressalta-se ainda, que o trabalho do enfermeiro, inserido nas instituições de saúde, é muitas vezes multifacetado, dividido e submetido a uma diversidade de cargos que geram desgaste. Assim considera-se a enfermagem como uma profissão estressante e esse fato se relaciona ao trabalho com pessoas que sofrem e requerem grande demanda de atenção (Vieira et al., 2013). 
Esse resultado também é corroborado por Murano, Santos \& Beresin, (2009) que apontaram em seu estudo, que a categoria enfermagem do CC vêm suportando grandes cargas trabalhistas muito elevadas e desproporcionais, períodos rotativos, manipulação de substâncias tóxicas e presença de condições favoráveis ao risco pertinente ao ambiente, o que leva a uma situação conhecida com grande carga de trabalho. Consequentemente a esta situação, o enfermeiro vivencia muita frustração e descontentamento no tocante à responsabilidade e exercício profissional, podendo resultar em transtornos físicos e psicológicos, afetando seu bem estar levando a um comprometimento de sua saúde.

Outros estudos, também vão ao encontro do que foi abordado nesta pesquisa, pois têm apontado ainda, como fatores prejudiciais à qualidade de vida no trabalho (QVT) dos enfermeiros: a remuneração ainda muito baixa, a manipulação de substâncias tóxicas e as condições que levam aos riscos pertinentes ao ambiente, além das dificuldades socioeconômicas enfrentadas por estes profissionais, pois muitos mantêm mais de um vínculo empregatício (Schmidt et al., 2013)

Sobre os transtornos físicos e psicológicos, o Tema 2 evidenciou as consequências do estresse ocupacional no trabalho do centro cirúrgico como sintomas físicos: dores musculares; cefaleia; irritabilidade; erupções cutâneas; dificuldades de concentração; má digestão e azia; problemas respiratórios e do aparelho urinário; resfriados prolongados, insônia, sensação de opressão no peito; náusea, palpitação e isolamento social; susceptibilidade; mau humor; desmotivação, refluxo gástrico, cansaço. Assim como, o desenvolvimento de doenças osteomuscular, infecciosas, hipertensão arterial, diarreia psicogênica, asma, inflamação do duodeno e fibromialgia (Soratto, et al, 2016; Tostes et al, 2017).

Além dos problemas físicos, evidenciou-se consequências emocionais, como maior exaustão emocional, ansiedade e estresse com efeitos no padrão de sono e de condição de saúde (Soratto, et al, 2016; Santos et al, 2019; Tostes et al, 2017; Carvalho, et al, 2018).

Outros estudos também apontam o estresse como o produto resultante do trabalho em bloco cirúrgico e apontado como o agente que afeta o fisico e o psicológico do trabalhador desta área, gerando processos desgastantes nas suas relações de trabalho e desempenho. O risco de morte é apontado como um dos principais motivos para o sofrimento e adoecimento emocional dos trabalhadores da saúde, que se perpetua em seu cotidiano. Sendo assim, há um gasto muito maior de energia por parte destes profissionais, para tentar manter-se equilibrado no seu sentir, pensar e agir (Semeniuk et al., 2012).

É certo que cada indivíduo possui uma maneira peculiar de defrontar diversos estressores e de expressar seus sentimentos, o que pode repercutir de maneira positiva ou negativa no processamento do trabalho, bem como na saúde, e por ser o centro cirúrgico um setor potencialmente estressante, aqueles que nele atuam estão constantemente sujeitos a apresentar algum tipo de manifestação de sofrimento e adoecimento físico e mental, inerentes às atividades desenvolvidas, tanto na área assistencial quanto de ensino (Sousa, 2011).

Ressalta-se que a grande continuidade de estresse trabalhista também é tida como um dos principais fatores geradores de transtornos mentais e de outras doenças, como, síndrome metabólica, da fadiga, de Burnout, crônica, desequilíbrios no sono (Rodrigues et al., 2010).

Sobre isso, a Organização Internacional do Trabalho (Oit, 2013), demonstra que, no mundo morrem dois milhões de trabalhadores a cada ano em decorrência de doenças ocupacionais. Esse número é seis vezes maior do que o de acidentes em seu local de trabalho e está relacionado em grande parte ao aumento do ritmo e da intensidade do trabalho para atender às necessidades e metas das organizações.

Entre os principais agravos a saúde dos trabalhadores sob estresse, destaca-se a ansiedade; a depressão; estresse ocupacional / laboral; Síndrome de Burnout; o suicídio e a exaustão emocional, que corroboram os encontrado neste estudo (Schmidt et al., 2010; Ratochinski et al., 2016; Meneghini, Paz \& Lautert, 2011).

Pontua-se que, a causa da exaustão psicoemocional está relacionada a uma redução ou ausência de energia vinculada ao processo de esgotamento emocional. Os sinais e sintomas podem ser físicos, psíquicos ou a junção de ambos. Estes 
profissionais já não encontram mais condições de prestar cuidados assistenciais aos pacientes, por falta de saúde e de energia (Rissardo \& Gasparino, 2013).

Outra consequência importante identificada neste estudo, foi a ansiedade que é um fenômeno que ora nos beneficia e ora nos prejudica, dependendo das circunstâncias ou intensidade, podendo tornar-se patológica, isto é, prejudicial ao nosso funcionamento psíquico (mental) e somático (corporal) (Brasil, 2015).

\section{Conclusão}

Este estudo teve como objetivo descrever as evidências científicas encontradas na literatura científica do período de 2015 a 2020, sobre os fatores estressores e suas consequências, para os profissionais de enfermagem que atuam no centro cirúrgico. Neste sentido, pôde-se elencar o ambiente fechado, a carga excessiva de trabalho, dinâmicas profissionais entre a equipe, falta de compromisso com o trabalho e os tabalhadores, falta de insumos e recursos materiais como agentes promotores de estresse no trabalho, além da baixa remuneração e valorização profissional, número insuficiente de profissionais qualificados, elevada demanda de cirurgias, condição dos pacientes, intercorrências e urgências; insuficiência de planejamento, turnos ou regime de plantões desgastantes e baixa autonomia.

E, como consequência do estresse ocupacional no centro cirúrgico esses profissionais apresentam principalmente: sintomas físicos gastrointestinais; dores; doenças osteomusculares; hipertensão; baixa imunidade; tendência maior a doenças infecciosas e respiratórias, além de comprometimento psicológico evidenciado por alta exaustão emocional; ansiedade; distúrbios no padrão de sono comprometimento do bem-estar.

Entretanto, o estudo apontou também, que a equipe que compõe os profissionais da enfermagem sentem satisfação com seu trabalho no centro cirúrgico, quando o ambiente é influenciado por investimento em conhecimento e reconhecimento profissional.

Ademais, esta pesquisa aponta que se faz necessário o uso das produções científicas para abrir caminho às intervenções nesta problemática, no intuito de garantir a melhora das condições trabalhistas da equipe de enfermagem no centro cirúrgico, pontuando que alguns dos resultados deste estudo já foram abordados em outras pesquisas, já há bastante tempo, porém notou-se que isto ainda não gerou melhoras significativas para esses trabalhadores.

Esta pesquisa pode servir como base para futuras discussões e estudos que possam ser debatidas nas academias durante a formação de enfermeiros, no intuito de contribuir com tais mudanças.

\section{Referências}

Andrade, M. C. M. \& Siqueira Júnior, A. C. (2017). Occupational stress in the mobile emergency care service. REME: Revista Mineira de Enfermagem, 18(2), $376-383$.

Brasil. Ministério da Saúde, 2015.

Cardoso, V. et al. (2019). Revisão sistemática de métodos mistos: método de pesquisa para a incorporação de evidências na enfermagem. Texto \& ContextoEnfermagem, 28. e20170279. Recuperado de: https://doi.org/10.1590/1980-265x-tce-2017-0279

Carvalho, A. D. M. B. et al. (2018). Qualidade de vida no trabalho da equipe de enfermagem do centro cirúrgico. Enfermagem em Foco, 9(3), 35-41.

Gomes, L. D. C. \& Dutra, K. E. (2014). O enfermeiro no gerenciamento do centro cirúrgico. Revista Eletrônica da Faculdade Metodista Granbery, (16),1-21.

Jacques, J. P. B. et al. (2015). Geradores de estresse para os trabalhadores de enfermagem de centro cirurgico Stress Generators for employees of surgical nursing center. Semina: Ciênc. Biol. Saúde, 36(1), 25-32, 2015.

Lima, T. F. da S. et al. (2021). Variáveis climáticas e sua relação com doenças de origem infecciosa: Uma revisão integrativa. Research, Society and Development, 10(2), e7910212126. https://doi.org/10.33448/rsd-v10i2.12126

Martins, F. Z.; \& Dall'agnol, C. M. (2016). Centro cirúrgico: desafios e estratégias do enfermeiro nas atividades gerenciais. Revista Gaúcha de Enfermagem, $37(4), 1-9$. 
Meneghini, F., Paz, A. A. \& Lautert, L. (2011). Fatores ocupacionais associados aos componentes da síndrome de burnout em trabalhadores de enfermagem. Texto e Contexto Enfermagem, 20(2), 225-233.

Moher, D., et al. (2009). Preferred reporting items for systematic reviews and meta-analyses: The PRISMA statement. PLoS Medicine. 6 (7).

Santos, W. M. dos, Secoli, S. R. \& Püschel, V. A. de A. (2018). A abordagem do Joanna Briggs Institute para revisões sistemáticas. Revista Latino-Americana de Enfermagem, 26. e3074.

Santos, R. M. A. \& Beresin, R. (2009). Qualidade de vida dos enfermeiros do centro cirúrgico. Einstein, 7(2), 152-160.

Organização Internacional do Trabalho (OIT). http://www.oitbrasil.org.br/sites/default/files/topic/gender/doc/safeday2013\%20final_1012.pdf

Passos, J. B., Silva, E. L. da \& Carvalho, M. M. C. de. (2010). Estresse no centro cirúrgico: uma realidade dos profissionais de enfermagem. Rev Pesq Saúde, 11(2), 35-38.

Paglione, N. L. et al. (2016). Satisfação profissional da equipe de enfermagem do centro cirúrgico em hospital público de média complexidade. Revista de Enfermagem UFPE On Line, 10(3), 1080-1087.

Pereira, A. S., et al. (2018). Metodologia da pesquisa científica. UFSM. https://repositorio.ufsm.br/bitstream/handle/1/15824/Lic_Computacao_MetodologiaPesquisa-Cientifica.pdf?sequence $=1$.

Puerto, J. C. et al. (2017). Uma nova contribuição para a classificação dos fatores que afetam os profissionais de enfermagem. Rev Latino Enfermagem. 25, 2895.

Ratochinski, C. M. W. et al. (2016). O Estresse em Profissionais de Enfermagem: Uma Revisão Sistemática. Revista Brasileira de Ciências da Saúde, 20(4), $341-346$.

Rissardo, M. P. \& Gasparino, R. C. (2013). Exaustão emocional em enfermeiros de um hospital público. Escola Anna Nery, 17(1), 128-132.

Semeniuk, A. P. et al (2012). Saúde Mental Da Equipe De Enfermagem De Centro Cirúrgico frente à morte. Revista SOBECC, 17(4), 48-56.

Schmidt, D. R. C. et al. (2009). Estresse ocupacional entre profissionais de enfermagem do bloco cirúrgico. Texto e Contexto-Enfermagem, 18(2), $330-337$.

Schmidt, D. R. C. et al. (2011). Ansiedade e depressão entre profissionais de enfermagem que atuam em blocos cirúrgicos. Rev Esc Enferm USP, 45(2), 48793.

Schmidt, D. R. C. et al. (2013). Qualidade de vida no trabalho e burnout em trabalhadores de enfermagem de Unidade de Terapia Intensiva. Revista Brasileira de Enfermagem, 66(1), 13-17.

Soares, C. B. et al. (2014). Revisão Integrativa versus Revisão Sistemática. Reme: Revista Mineira de Enfermagem, 12(4), $758-764$.

Soares, D. L. et al. (2017). Qualidade de vida dos profissionais atuantes no centro cirúrgico. Psicologia e Saúde em Debate, 3(2), 159-170.

Sousa, F. M. S. (2011). Condições de trabalho de ambiente cirúrgico e a saúde dos trabalhadores de enfermagem. Dissertação (Mestrado em Enfermagem) Programa de Pós-Graduação em Enfermagem, Universidade do Estado do Rio de Janeiro.

Stumm, E. M. F. et al. (2010). Estressores e sintomas de estresse vivenciados por profissionais em um centro cirúrgico. REME rev. min. enferm, 54-63.

Teixeira, E. et al. (2014). RIL métodos de revisão. Revista de Enfermagem da UFPI, 2(5), 3.

Tostes, M. F. D. P. et al. (2017). Dualidade entre satisfação e sofrimento no trabalho da equipe de enfermagem em centro cirúrgico. Revista SOBECC, 22(1), 3-9.

Ueno, L. G. S. et al. (2017). Estresse ocupacional: estressores referidos pela equipe de enfermagem. Rev. Enferm. UFPE Online, 11(4), $1632-1638$.

Ursi, E. S. \& Galvão, C. M. (2015). Avaliação das escalas de risco para úlcera por pressão em pacientes críticos: uma coorte prospectiva. Acta Paul Enferm, $23(1), 28-35$.

Versa, G. L. G. da S. et al. (2012). Estresse ocupacional: avaliação de enfermeiros intensivistas que atuam no período noturno. Revista Gaúcha de Enfermagem, 33(2), 78-85.

Vieira, F. D. S. et al. (2013). Estresse: fatores desencadeadores no exercício profissional de enfermeiros. Revista de Enfermagem da UFPI, $2,55-59$. 